\title{
Primary Decomposition of Powers of the Prime Ideal of a Numerical Semigroup Ring
}

\author{
Ralf Fröberg ${ }^{1}$
}

To Le Tuan Hoa on his sixtieth birthday

Received: 13 April 2018 / Revised: 11 August 2018 / Accepted: 6 November 2018 /

Published online: 11 January 2019

(C) The Author(s) 2019

\begin{abstract}
Let $R=k\left[t^{n_{1}}, \ldots, t^{n_{s}}\right]=k\left[x_{1}, \ldots, x_{s}\right] / P$ be a numerical semigroup ring and let $P^{(n)}=$ $P^{n} R_{P} \cap R$ be the symbolic power of $P$ and $R_{S}(P)=\oplus_{i \geq 0} P^{(n)} t^{n}$ the symbolic Rees ring of $P$. It is hard to determine symbolic powers of $P$; there are even non-Noetherian symbolic Rees rings for 3-generated semigroups. We determine the primary decomposition of powers of $P$ for some classes of 3-generated numerical semigroups.
\end{abstract}

Keywords Primary decomposition $\cdot$ Symbolic power $\cdot$ Numerical semigroup ring

Mathematics Subject Classification (2010) Primary 13A30 · Secondary 13 H10

\section{Introduction}

If $I$ is an ideal in a Noetherian ring $R$, then the subring $R(I)=R[I t]$ of $R[t]$ is called the Rees ring of $I$. This was introduced by Rees, in his proof of the Artin-Rees lemma. If $P$ is a prime ideal, then the primary decomposition of $P^{n}$ always contains a $P$-primary component, it is $R=k\left[t^{n_{1}}, \ldots, t^{n_{s}}\right]=k\left[x_{1}, \ldots, x_{n}\right] / P$ and is called the symbolic $n$th power of $P$. Cowsik [1] asked if the symbolic Rees algebra $R_{S}(P)=R\left[P t, P^{(2)} t^{2}, P^{(3)} t^{3}, \ldots\right]$ is always Noetherian. This was shown not to be true by Roberts [7, 8]. There are even counterexamples when $R=k\left[t^{n_{1}}, t^{n_{2}}, t^{n_{3}}\right]=k[x, y, z] / P$. Goto-Nishida-Watanabe [2] showed that for $n \geq 4$ then $k\left[t^{7 n-3}, t^{(5 n-2) n}, t^{8 n-3}\right]$ does not have a finitely generated symbolic Rees algebra if $\operatorname{char}(k)=0$. Their smallest counterexample is $k\left[t^{25}, t^{72}, t^{29}\right]=$ $k[x, y, z] /\left(x^{11}-y z^{7}, y^{3}-x^{4} z^{4}, z^{11}-x^{7} y^{2}\right)$. Hochster [4] has shown that $P^{(n)}=P^{n}$ for all $n$ if $k\left[x_{1}, \ldots, x_{s}\right] / P$ is a complete intersection. Huneke [5] has shown that if $P$ is a prime ideal of height 2 in a 3 -dimensional ring, then the opposite holds.

Ralf Fröberg

ralff@math.su.se

1 Department of Mathematics, Stockholm University, Stockholm, Sweden 


\section{Numerical Semigroup Rings}

If $R=k\left[t^{n_{1}}, \ldots, t^{n_{s}}\right]$, we map $k\left[x_{1}, \ldots, x_{s}\right]$ to $k[t]$, by $x_{i} \mapsto t^{n_{i}}$. Then $R \simeq$ $k\left[x_{1}, \ldots, x_{s}\right] / P$, and $P$ is a prime ideal since $R$ is a domain. In the case of numerical semigroup rings, $R$ is 1-dimensional, so $P^{(n)}=P^{n}$ or $P^{(n)} \cap Q$, where $Q$ is $\left(x_{1}, \ldots, x_{s}\right)$ primary. If $P^{(n)}=P^{n}$ for all $n$, i.e., if the semigroup ring is a complete intersection, then $R_{S}(P)=R(P)$ is Noetherian and $P^{n}$ is $P$-primary. Kunz [6] has shown that the semigroup ring of $S$ is Gorenstein if and only if the semigroup $S$ is symmetric, i.e., if for some $D$, we have $n \in S$ if and only if $D-n \notin S$.

\section{3-Generated Numerical Semigroups}

In the sequel, we mean numerical semigroup when we write semigroup. A 3-generated semigroup is Gorenstein if and only if it is a complete intersection. This is so, since if we factor with a generator, we get a ring of embedding dimension 2 . The factor ring is Gorenstein (complete intersection) if and only if the semigroup ring is Gorenstein (complete intersection). For a ring of embedding dimension 2, the concepts are equivalent. If the semigroup is generated by 3 elements, and is not a complete intersection, then $R=k\left[t^{n_{1}}, t^{n_{2}}, t^{n_{3}}\right] \simeq k[x, y, z] / P$ where $P$ is generated by the three $2 \times 2$-minors of a matrix (the relation matrix)

$$
\left(\begin{array}{lll}
x^{a_{1}} & y^{b_{1}} & z^{c_{1}} \\
z^{c_{2}} & x^{a_{2}} & y^{b_{2}}
\end{array}\right)
$$

Herzog and Ulrich [3] have shown that $R_{S}(P)=R\left[P t, P^{(2)} t^{2}\right]$ if and only if $a_{1}=a_{2}, b_{1} \leq$ $b_{2}, c_{1} \geq c_{2}$ (or a permutation). Huneke [5] has shown that if $P$ is a 2-dimensional prime in a 3-dimensional ring, then $P^{(2)} / P^{2}$ is generated by one element $\Delta$. Schenzel [9] has, in the case of a 3-generated semigroup, determined $\Delta$. The result is, if $a_{1} \leq a_{2}, b_{1} \geq b_{2}, c_{1} \geq c_{2}$, in particular this holds if $R_{S}(P)=R\left[P t, P^{(2)} t^{2}\right]$ according to the result of Herzog and Ulrich,

$$
\Delta=\left|\begin{array}{ccc}
x^{a_{1}} & y^{b_{1}} & z^{c_{1}} \\
z^{c_{2}} & x^{a_{2}} & y^{b_{2}} \\
y^{b_{1}} & x^{a_{2}-a_{1}} y^{b_{1}-b_{2}} z^{c_{2}} & y^{a_{1}} z^{c_{1}-c_{2}}
\end{array}\right| .
$$

He also showed that $\left(x^{a_{1}}, y^{b_{2}}, z^{c_{2}}\right) \Delta \in P^{2}$.

In the other case, $a_{1} \geq a_{2}, b_{1} \geq b_{2}, c_{1} \geq c_{2}$, there is a similar result:

$$
\Delta=\left|\begin{array}{ccc}
x^{a_{1}} & y^{b_{1}} & z^{c_{1}} \\
z^{c_{2}} & x^{a_{2}} & y^{b_{2}} \\
x^{a_{1}-a_{2}} & y^{b_{1}-b_{2}} z^{c_{1}} & x^{a_{1}} z^{c_{1}-c_{2}}
\end{array}\right|
$$

and $\left(x^{a_{2}}, y^{b_{2}}, z^{c_{2}}\right) \Delta \in P^{2}$. If $R_{S}(P) \neq R\left[P t, P^{(2)} t^{2}\right]$, we can only determine the primary decomposition of $P^{2}$, but if $R_{S}(P)=R\left[P t, P^{(2)} t^{2}\right]$, we will construct the primary decomposition of $P^{n}$ for all $n$ in the following cases: if the semigroup is generated by an arithmetic sequence, or if it is generated by $a<b<c$ with $c-a \leq 4$, or if the multiplicity is $\leq 4$.

Theorem 1 Suppose that $R=k\left[t^{a}, t^{b}, t^{c}\right]=k[x, y, z] / P$ is not a complete intersection. Then $P^{2}=\left((\Delta)+P^{2}\right) \cap\left(\left(z^{c_{2}}\right)+P^{2}\right)$ is a primary decomposition. If furthermore $R_{S}(P)=$ $R\left[P t, P^{(2)} t^{2}\right]$, then $P^{2 n}=\left(P^{(2)}\right)^{n} \cap\left(\left(z^{n c_{2}}\right)+P^{2 n}\right)$ and $P^{2 n+1}=P\left(P^{(2)}\right)^{n} \cap\left(\left(z^{n c_{2}}\right)+P^{2 n}\right)$. 
Proof Since $P^{(2)}=(\Delta)+P^{2}$ and since $\left(z^{c_{2}}\right)+P^{2}$ is $(x, y, z)$-primary, it suffices to note that $\left(z^{c_{2}}\right) \cap(\Delta)=z^{c_{2}} \Delta \subseteq P^{2}\left(\left[9\right.\right.$, Theorem 10.3]) to see the first statement. If $R_{S}(P)=$ $R\left[P t, P^{(2)} t^{2}\right]$, then $P^{(2 n)}=\sum_{i=0}^{n}\left(P^{(2)}\right)^{i} P^{2 n-2 i}=\left(P^{(2)}\right)^{n}$ since $P^{2} \subseteq P^{(2)}$. In the same way, we see that $P^{(2 n+1)}=P P^{(2 n)}$. Finally, $\left(z^{n c_{2}}\right) \cap P^{(2 n)}=\left(z^{n c_{2}}\right) \cap\left((\Delta)+\left(P^{2}\right)^{n}\right) \subseteq P^{2 n}$ since $z^{c_{2}} \Delta \subseteq P^{2}$.

The remaining part is a search for examples when $R_{S}(P)=R\left[P t, P^{(2)} t^{2}\right]$.

\subsection{Arithmetic Sequences}

Now suppose that the semigroup is generated by $m, m+d, m+2 d, \operatorname{gcd}(m, m+d, m+2 d)=$ 1. The semigroup is symmetric (so the semigroup ring is a complete intersection) if $m$ is even and $d$ odd. Otherwise, the relation matrix is

$$
\left(\begin{array}{ccc}
x^{k+d} & y & z \\
z^{k} & x & y
\end{array}\right) .
$$

Thus, according to the theorem by Herzog and Ulrich $R_{S}(P)=R\left[P t, P^{(2)} t^{2}\right]$. We use this result also below to see when $R_{S}(P)=R\left[P t, P^{(2)} t^{2}\right]$.

Theorem 2 Let $R=k\left[t^{m}, t^{m+d}, t^{m+2 d}\right]=k[x, y, z] / P$ be nonsymmetric. Then the primary decomposition is $P^{2 n}=\left((\Delta)+P^{2}\right)^{n} \cap\left(\left(z^{n}\right)+P^{2}\right)^{n}$ and $P^{2 n+1}=\left(P(\Delta)+P^{2}\right)^{n} \cap$ $\left(\left(z^{n}\right)+P^{2}\right)^{n}$.

\subsection{Semigroups Generated by $a<b<c, c-a \leq 4$}

If the semigroup is not generated by an arithmetic sequence, the generators are $m, m+$ $1, m+3$ or $m, m+1, m+4$ or $m, m+2, m+3$ or $m, m+3, m+4$.

If the semigroup is generated by $m, m+1, m+3$, it is symmetric if $m \equiv 0(\bmod 3)$.

If $m=3 k+1$ the relation matrix is

$$
\left(\begin{array}{ccc}
x^{k} & y & z \\
z^{k} & x^{2} & y^{2}
\end{array}\right)
$$

and $R_{S}(P) \neq R\left[P t, P^{(2)} t^{2}\right]$ for all $k$.

If $m=3 k+2$ the relation matrix is

$$
\left(\begin{array}{ccc}
x^{k+1} & y^{2} & z \\
z^{k} & x^{2} & y
\end{array}\right)
$$

and $R_{S}(P) \neq R\left[P t, P^{(2)} t^{2}\right]$ unless $k=1$.

If the semigroup is generated by $m, m+1, m+4$, it is symmetric if $m \equiv 0(\bmod 4)($ and if $m=5$ ).

If $m=4 k+1, k \geq 2$, the relation matrix is

$$
\left(\begin{array}{ccc}
x^{k-1} & y & z \\
z^{k} & x^{3} & y^{3}
\end{array}\right)
$$

and $R_{S}(P) \neq R\left[P t, P^{(2)} t^{2}\right]$.

If $m=4 k+2, k \geq 2$, the relation matrix is

$$
\left(\begin{array}{ccc}
x^{k} & y^{2} & z \\
z^{k} & x^{3} & y^{2}
\end{array}\right)
$$


and $R_{S}(P)=R\left[P t, P^{(2)} t^{2}\right]$ if and only if $k \geq 3$ and $k=1$.

If $m=4 k+3$, the relation matrix is

$$
\left(\begin{array}{ccc}
x^{k+1} & y^{3} & z \\
z^{k} & x^{3} & y
\end{array}\right)
$$

and $R_{s}(P)=R\left[P t, P^{(2)} t^{2}\right]$ only if $k=1$ or $k=2$.

If the semigroup is generated by $m, m+2, m+3$, it is symmetric if $m \equiv 0(\bmod 3)$ (and if $m=4$ ).

If $m=3 k+1$, the relation matrix is

$$
\left(\begin{array}{ccc}
x^{k+1} & y^{2} & z^{2} \\
z^{k-1} & x & y
\end{array}\right)
$$

and $R_{S}(P) \neq R\left[P t, P^{(2)} t^{2}\right]$ for all $k$.

If $m=3 k+2$ the relation matrix is

$$
\left(\begin{array}{ccc}
x^{k} & y^{2} & z^{2} \\
z^{k} & x & y
\end{array}\right)
$$

and $R_{S}(P) \neq R\left[P t, P^{(2)} t^{2}\right]$ for all $k$.

If the semigroup is generated by $m, m+3, m+4$, it is symmetric if $m \equiv 0(\bmod 4)$ (and if $m=6$ or $m=9$ ).

If $m=4 k+1, k \geq 2$, the relation matrix is

$$
\left(\begin{array}{ccc}
x^{k+1} & y^{3} & z^{3} \\
z^{k-1} & x & y
\end{array}\right)
$$

and $R_{S}(P) \neq R\left[P t, P^{(2)} t^{2}\right]$.

If $m=4 k+2, k \geq 2$, the relation matrix is

$$
\left(\begin{array}{ccc}
x^{k+1} & y^{2} & z^{3} \\
z^{k-1} & x & y^{2}
\end{array}\right)
$$

and $R_{S}(P)=R\left[P t, P^{(2)} t^{2}\right]$ if and only if $k \geq 4$.

If $m=4 k+3$ the relation matrix is

$$
\left(\begin{array}{ccc}
x^{k+1} & y & z^{3} \\
z^{k} & x & y^{3}
\end{array}\right)
$$

and $R_{S}(P)=R\left[P t, P^{(2)} t^{2}\right]$ only if $k=3$.

For $m=5$ the relation matrix is

$$
\left(\begin{array}{lll}
x^{2} & y^{2} & z \\
z & x^{3} & y
\end{array}\right)
$$

and $R_{S}(P)=R\left[P t, P^{(2)} t^{2}\right]$.

Theorem 3 If the semigroup is generated by $a<b<c, c-a \leq 4$, not symmetric, and $a, b, c$ not an arithmetic sequence, then $R_{S}(P)=R\left[P t, P^{(2)} t^{2}\right]$ if and only if the generators are $5,6,8$ or $6,7,10$ or $15,18,19$ or $7,8,11$ or $11,12,15$ or $15,18,19$ or $4 k+2,4 k+3,4 k+6, k \geq 3$ or $4 k+2,4 k+5,4 k+6, k \geq 4$.

\subsection{Semigroups of Multiplicity 3}

We note that if the semigroup is of multiplicity $\leq 4$, then Huneke [5] has shown that the symbolic Rees ring is Noetherian. Suppose that the semigroup is generated by $3,3 k+1,3 l+$ 
2. In order to have a 3-generated semigroup, we must have $l \leq 2 k$ and $k \leq 2 l+1$. The semigroup is never symmetric. The relation matrix is

$$
\left(\begin{array}{ccc}
x^{2 l-k+1} & y & z \\
z & x^{2 k-l} & y
\end{array}\right)
$$

and $R_{S}(P)=R\left[P t, P^{(2)} t^{2}\right]$.

\subsection{Semigroups of Multiplicity 4}

If a 3-generated semigroup has multiplicity 4 and is not symmeteric, it has generators $4,4 k+$ $1,4 l+3$. If $k>l$ the relation matrix is

$$
\left(\begin{array}{ccc}
x^{3 l-k+2} & y & z^{2} \\
z & x^{2 k-2 l-1} & y
\end{array}\right)
$$

and $R_{S}(P)=R\left[P t, P^{(2)} t^{2}\right]$ if and only if $5 l-3 k+3 \leq 0$. If $k \leq l$, the relation matrix is

$$
\left(\begin{array}{ccc}
x^{2 l-2 k+1} & y & z \\
z & x^{3 k-l} & y^{2}
\end{array}\right)
$$

and $R_{S}(P)=R\left[P t, P^{(2)} t^{2}\right]$ if and only if $3 l-5 k+1 \geq 0$.

Theorem 4 If the semigroup has multiplicity 3 , then $R_{s}(P)=R\left[P t, P^{(2)} t^{2}\right]$. If the multiplicity is 4 and not symmetric, it is generated by $4,4 k+1,4 l+3$, and $R_{S}(P)=$ $R\left[P t, P^{(2)} t^{2}\right]$ if and only if $k>l$ and $5 l-3 k+3 \leq 0$ or if $k \leq l$ and $3 l-5 k+1 \geq 0$.

Open Access This article is distributed under the terms of the Creative Commons Attribution 4.0 International License (http://creativecommons.org/licenses/by/4.0/), which permits unrestricted use, distribution, and reproduction in any medium, provided you give appropriate credit to the original author(s) and the source, provide a link to the Creative Commons license, and indicate if changes were made.

Publisher's Note Springer Nature remains neutral with regard to jurisdictional claims in published maps and institutional affiliations.

\section{References}

1. Cowsik, R.: Symbolic powers and the number of defining equations. Algebra and its Applications, Lecture Notes in Pure and Appl. Math. 9, 13-14 Dekker New York (1984)

2. Goto, S., Nishida, K., Watanabe, K.: Non-Cohen-Macaulay symbolic blow-ups for space monomial curves and counterexamples to Cowsik's question. Proc. Am. Math. Soc. 120(2), 383-392 (1994)

3. Herzog, J., Ulrich, B.: Self-linked curve singularities. Nagoya Mat. J. 120, 129-153 (1990)

4. Hochster, M.: Criteria for equality of ordinary and symbolic powers of primes. Math. Z. 133, 53-65 (1973)

5. Huneke, C.: Primary components and integral closures of ideals in 3-dimensional regular local rings. Math. Ann. 275, 617-635 (1986). c.i.

6. Kunz, E.: Almost complete intersections are not Gorenstein rings. J. Algebra 28, 111-115 (1974)

7. Roberts, P.: A prime ideal in a polynomial ring whose symbolic blow-up is not Noetherian. Proc. Am. Math. Soc. 94, 589-592 (1985)

8. Roberts, P.: An infinitely generated symbolic blow-up in a power series ring and a new counterexample to Hilbert's fourteenth problem. J. Algebra 132, 461-473 (1990)

9. Schenzel, P.: Examples of Noetherian symbolic blow-up rings. Rev. Roumaine Math. Pures Appl. 33, 375-383 (1988) 